Relations industrielles

Industrial Relations

\title{
Union Power and the Public Interest, by Emerson P. Schmidt, Los Angeles, Calif., Nash Publishing Corp., 1973, 204 pp.
}

\section{Jean Boivin}

Volume 29, numéro 4, 1974

URI : https://id.erudit.org/iderudit/028565ar

DOI : https://doi.org/10.7202/028565ar

Aller au sommaire du numéro

Éditeur(s)

Département des relations industrielles de l'Université Laval

ISSN

0034-379X (imprimé)

1703-8138 (numérique)

Découvrir la revue

Citer ce compte rendu

Boivin, J. (1974). Compte rendu de [Union Power and the Public Interest, by Emerson P. Schmidt, Los Angeles, Calif., Nash Publishing Corp., 1973, 204 pp.] Relations industrielles / Industrial Relations, 29(4), 884-885.

https://doi.org/10.7202/028565ar

Tous droits réservés @ C Département des relations industrielles de l'Université Laval, 1974
Ce document est protégé par la loi sur le droit d'auteur. L'utilisation des services d'Érudit (y compris la reproduction) est assujettie à sa politique d'utilisation que vous pouvez consulter en ligne.

https://apropos.erudit.org/fr/usagers/politique-dutilisation/ 
ment, mais il pourrait constituer le canevas d'une étude beaucoup plus approfondie, en particulier en ce qui concerne les sources idéologiques où ont puisé leurs dirigeants.

Quant à l'étude de Louis Maheu, elle cherche à établir les fondements du syndicalisme catholique au Québec. L'idée centrale qu'on en retient, c'est que le clergé à l'époque, face aux transformations de la vie économique, principalement la naissance de l'industrie ou d'une forme nouvelle de l'industrie, a voulu par ce moyen s'assurer que la société ne changerait pas ou ne devrait pas changer et qu'il fallait, par conséquent, réagir contre la tendance «au cloisonnement et à l'isolement des ouvriers industriels et urbains 》, ce qui allait «à l'encontre des traditions séculaires de la communauté et du nous collectif homogène » en tentant de «sacraliser» le monde du travail et d'imposer un type de société médiéval.

Ces études disparates, peut-être même discordantes sont-elles un apport valable à l'histoire du mouvement syndical au Québec? Si, d'une part, on peut affirmer qu'elles sont des pièces utiles, une fois travaillées à l'organisation d'un ensemble, on ne saurait dire, d'autre part, qu'elles constituent une synthèse historique, car, comme l'écrivait Robert Lenoble, "le rôle de l'historien est de comprendre le sens que prend la causalité dans chaque système », ce qui ne va pas jusque-là.

Ministère du travail et de la

\section{André ROY}

\section{main-d'oeuvre}

Québec

Union Power and the Public Interest, by Emerson P. Schmidt, Los Angeles, Calif., Nash Publishing Corp., 1973, $204 \mathrm{pp}$.

Ce livre est un plaidoyer systématique contre les syndicats. L'auteur, sur la base de l'expérience américaine, tente de démontrer que le pouvoir monopolistique exercé par les syndicats sur la main-d'oeuvre est la cause des principaux maux affligeant l'économie. Plus précisément, il affirme que parce que les activités syndicales ont été protégées par des lois spéciales dans les années 1930 (notamment la loi Norris-La-Guardia et le Wagner Act), il en est résulté un abus de pouvoir qui a conduit à des grèves et à de la violence pour imposer des taux de traitements supérieurs à l'accroissement de la productivité. Cette escalade des salaires, selon l'auteur, a ensuite entraîné une élévation des prix et une augmentation du chômage de sorte qu'ultimement ce sont les non syndiqués et les consommateurs en général qui ont dû en subir les conséquences négatives.

Ce livre fait partie d'une collection intitulée \& The Principles of Freedom Committee » dont les principaux collaborateurs se sont fixés comme objectifs de défendre les «valeurs fondamentales de l'Occident» (voir préface) telles la liberté individuelle, la propriété privée, la libre entreprise, etc. Il s'agit donc d'une littérature spécifiquement engagée dans la défense d'une cause ou d'une idéologie et il est bon de s'en rappeler lorsque l'on fait la lecture du volume.

Comme corollaire à la critique du syndicalisme on $y$ retrouve également une dénonciation du rôle joué par l'Etat tant dans les relations patronales-ouvrières que dans l'économie en général. L'auteur critique le gouvernement qui tolère que des fonds de grève énormes soient constitués pour permettre aux travailleurs de tenir le coup lors d'un conflit avec leur employeur. Il s'attaque également au système qui prévaut dans certains Etats où les grévistes sont en mesure de recevoir des prestations d'assurance-chômage ou d'assistance sociale après un certain nombre de semaines. Enfin, Emerson P. Schmidt porte un jugement sévère contre les lois du salaire minimum qui sont, selon lui, la cause directe du taux de chômage élevé chez les jeunes, les non qualifiés et les minorités sociales.

On dénote une certaine contradiction dans la position de l'auteur lorsqu'à la fin du volume il conclut qu'après tout, la syndicalisation n'a profité qu'à une infime minorité de dirigeants ou au mieux, à une fraction de travailleurs qui, de toute façon, aurait quand même retiré des avantages particuliers sans la syndicalisation. Si cette affirmation s'avérait exacte, comment le professeur Schmidt peut-il simultanément affirmer que les syndicats sont responsables de tous les problèmes économiques qu'il a décrits antérieurement? La principale raison expliquant cette contradiction 
vient du fait que l'hypothèse de travail de l'auteur repose davantage sur ses préjugés personnels que sur des faits scientifiquement vérifiés. On ne retrouve aucune allusion aux études pourtant nombreuses ayant déjà été faites dans le but de mesurer l'impact économique des syndicats et de la négociation collective. Le professeur Schmidt préfère plutôt s'en remettre (et il le fait fréquemment) à des résultats de sondages d'opinion publique dans lesquels la majorité silencieuse affiche ouvertement ses convictions viscéralement anti-syndicales et qui rejoignent celles de l'auteur.

Bref, Union Power and the Public Interest ne présente aucun intérêt particulier si ce n'est pour les irréductibles anti-syndicaux et pour les économistes néo-classiques qui ont encore la nostalgie du modèle de concurrence parfaite.

Université Laval

\section{Jean BOrVIN}

\section{Les travailleurs du coton au Québec -} 1900 - 1915, par Jacques Rouillard, Montréal, Les Presses de l'Université du Québec, 1974, 152 pp.

C'est une courte étude très captivante que cette histoire économique et sociale de l'industrie du textile coton au Québec de 1900 à 1915, d'autant plus qu'il s'agit d'une période et d'un domaine assez peu connus.

Le travail est divisé en quatre chapitres distincts : l'entreprise, les travailleurs, le syndicalisme et les grèves. On peut s'étonner que l'auteur se soit limité à une période si courte, mais on ne saurait imaginer combien il a pu réunir dans une monographie restreinte dans le temps une masse aussi impressionnante de faits et de statistiques.

Dans le premier chapitre, l'auteur retrace l'évolution de l'industrie du textile coton du Québec depuis l'origine jusqu'à 1915 faisant notamment ressortir qu'elle eut un taux de croissance très élevé et qu'elle fut caractérisée, phénomène nouveau, par la concentration de la main-d'oeuvre en un petit nombre d'établissements.

Il faut surtout s'arrêter au deuxième chapitre qui présente en quelque sorte le travailleur du textile. Il y est traité de la composition de la main-d'oeuvre formée à l'époque d'un fort pourcentage de femmes et d'enfants en bas ge. On y voit, par exemple, nombre d'enfants travaillant dans cette industrie souvent poussés par leurs parents, également employés, qui touchent des salaires extrêmement bas, quoique le taux de participation des jeunes a tendance à décroître au fur et à mesure que les années passent. Au Québec, une loi connue sous le nom d'Acte des manufactures de Québec prohibe le travail des filles et des garçons de moins de 14 ans, mais cette loi est inopérante. Ce n'est que sous la pression des témoignages rendus devant la Commission royale d'enquête sur le capital et le travail que le gouvernement accepte enfin de nommer des inspecteurs.

Les conditions de travail sont mauvaises. L'auteur décrit ainsi la journée de travail d'un employé du textile en 1908: "Levé à cinq heures, l'ouvrier commence à travailler à six heures et quart. L'usine ouvre ses portes un quart d'heure plus tôt, puis la barrière est refermée aussitôt à clé, et ouverte vingt minutes plus tard pour les retardataires qui verront leurs salaires amputés de l'équivalent d'une demi-heure de travail. Après six heures de travail, la cloche d'arrêt sonne pour l'heure du dîner... La demi-heure écoulée, la cloche sonne à nouveau la reprise du travail qui dure jusqu'à cinq heures et trente sans interruption. L'ouvrier regagne alors son foyer pour prendre quelques heures de repos avant le dur labeur du lendemain. Des milliers de travailleurs, de novembre à mars, entrent donc à l'usine avant le jour pour n'en ressortir qu'à la nuit. Le seul répit survient en période de ralentissement de la production alors que les heures et les journées de travail, de même que les salaires, sont réduits $\gg$.

La semaine de travail est donc de dix heures par jour six jours par semaine et elle se passe dans une atmosphère chaude et humide au milieu du bruit sous la surveillance de contremaîtres d'une grande sévérité. Les accidents sont nombreux et avant 1909 aucune législation n'existe qui puisse atténuer quelque peu les conséquences dommageables de ces accidents.

D'autre part, les salaires, s'ils ne sont pas tellement inférieurs à ceux qui sont payés en Ontario dans cette industrie, par exemple, restent fort bas. Le travail 